\title{
Surgimiento y manifestación de las necesidades de información en los investigadores
}

\author{
Juan José Calva González \\ Investigador del CentroUniversitario de In- \\ vestigaciones Bibliotecológicas de la \\ UNAM. Torre II de Humanidades, piso 12, \\ CiudadUniversitaria, México D.F. tel: 623 \\ 0358 \\ E-mail:jjcg@servidor.unam.mx
}

RESUMEN

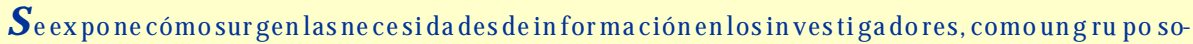
cial, partien do del pun to de vis ta de que és tas son un fe nó me no re pe ti ti vo que se pre sen ta en ellos. Al ser un fe nó me no que apa re ce con ti nua men te, en ton ces de ben exis tir prin ci pios que intervengan en la apa ri ción de éste. Asi mis mo se pre sen ta un mo de lo ten ta ti vo para ex pli car talfenómeno en el cual se in clu yen los ele men tos que in ter vie nen y cómo se re la cio nan para in fluir s o bre las necesi da des de in for ma ción y su ma ni fes ta ción a tra vés del com porta mien to in for ma ti vo (búsque da de información), que presentanlosinvestigadores.

ABSTRACT

$\boldsymbol{T}$ his pa per pre sents the for ming of in for ma tion ne eds in re sear chers, as a so cial group, starting from the viewpoint that these ne eds are a re pe ti ti ve phe no me non pre sen ted in them. By be ing a phe no me non that con ti nous ly appears, it should them exist prin ci ples that in ter ve ne in the appearing of it, in the same way is presented, a tentative model for explaining such phenomenon, in which are in clu ded the ele ments that in ter ve ning, how they are re la ted among them, and how they im pact on in for ma tion ne eds and their ma ni fes ta tion through the in for ma tion beha vior (information seeking behavior) showed by researchers.

\section{INTRODUCCIÓN}

$\mathrm{L}$ as necesi dades de infor ma ción pue den serconsideradas como una de las partes centrales en la teoría bibliotecológica y de las cien cias de la in for ma ción, pues to que las actividades bibliotecariasy de información se sustentan en satisfacer las necesidades que tienen las personas.

De tec taro de terminarlas necesidades de información, así como su satisfacción, puede ser considerado como uno de los pro pó si tos bá si cos de las uni da des de información, llámense bibliotecas, hemerotecas, centros de documentación, centros de información, etcétera.
Pero, las ne ce si da des de in for ma ción deben ser vistas como un fenómeno que se repite infinidad de veces en nuestra socie dad ac tual y que apa re ce en las per sonas como en tes in di vi dua les o cuan do están en grupo (por ejemplo, en las organizaciones).

Como un fenómeno que es repetitivo debe ac tuar bajo cier tos prin ci pios, por lo cual podemos suponer que pueden ser par te de una teo ría. Para des cu brir las variables y las interrelaciones que puedan existir entre ellas, en dicho suceso, éste debe ser estudiado con la rigurosidad como cualquier otro fenómeno físico, químico o biológico.

En la pre sen ta ción de este tra ba jo se pretenden bosquejar los elementos que inter vie nen en la apa ri ción de este fe nó meno, en el caso particular de los investigadores, y su in fluen cia en la ma nifestación de las necesidades de información a tra vés del com porta mien to in formativo.

Asimismo se expone un modelo tentativo de cómo pueden ser ubicados los elementos que intervienen en el estudio de las necesidades de información. Dicho modelo no es perfecto y puede ser am- 
pliado o corregido, pero su finalidad es empezar a esquematizar los elementos que puedan formar parte de una teoría acerca de las necesidades de información.

\section{LA MANIFESTACIÓN DE LAS NECESIDADES DE INFORMACIÓN}

El comportamiento de los individuos está motivado por una necesidad. Según la teoría de la Jerarquía de Necesidades de Maslow, cuando una persona tiene una necesidad cualquiera que sea ésta impulsa o mo tivaal in di vi duo a pre sen tar un com portamien to particular, porejemplo, cuan do se pre sen ta en la per so na una necesi dadfi sio ló gi ca como el ham bre, el comportamiento del individuo estará dirigido a satisfacer tal necesidad.

Lo mismo ocurre cuando una persona tiene una necesidad de información, presen ta un com por ta mien to parasa tis facerla. Este comportamiento de búsqueda de información es el que observamos cuando un in di vi duo, por ejem plo, en tra a una biblioteca y plantea una pregunta al servicio de consul ta o se dirige direc ta mente al catálogo a buscar la información que necesita.

Todo comportamiento, podemos decir, se manifiesta en tres áreas: mente y habla; cuerpo; y acción. ${ }^{1}$

Lanecesidad de in for ma ción que presentaelindividuo, primeramente, es percibi da por el cerebro de la persona, quien se manifiesta por medio del habla, proceso que pertenece a la primera área.

En seguida, el cerebro manda señales a través del sistema nervioso del organismo, por lo tanto se manifiesta una señal en elcuer podel in di viduo, posiblemente con una señal no verbal. Lo cual puede equivaler a la segunda área.

Y una ac ción, la ter ce ra área, es cuan do el individuo hace algo para satisfacer una ne ce si dad que tie ne, por ejem plo, di ri girse a la biblioteca pública y bus car en sus catálogos.
Es así como todo com por ta mien to, ya sea que se trate de un procesodesarrolladoexterior o interiormente en el pensamiento del in di viduo, se ma ni fies ta con el fin de que la persona se adapte o readapte al medio que lo rodea.

El in di vi duo sólo ma ni fies ta un com portamiento si siente una necesidad, es decir, si se rompe momentáneamente el equi li brio en tre el me dio y su or ga nis mo, entonces la acción que manifiesta dicha persona tiende a restablecerelequilibrio para readaptarsu orga nis mo al am bien te en que se encuentra. ${ }^{2}$

Por lo an te rior, el in di vi duo está in flui do por la interrelación que existe entre los sistemas: fisiológico, emocional, cognitivo, y social, los cuales determinan su necesidad de información y por lo tanto el comportamiento que sigue en la búsqueda de información para satisfacer su necesidad.

En ton ces todo com por ta mien to está de limi ta do porfac to res in di viduales - fi sioló gi cos, emo cionales, cog nitivos-y sociales, únicamente separables con fines descriptivos, ya que éstos se combinan y condicionan entrañablemente. ${ }^{3} \mathrm{El}$ comportamiento puede ser comprendido en función de la necesidad que presenta la persona dentro del contexto en el cual ocurre.

Podemos decir que los patrones de búsque da de in for mación es tán re laciona dos con una necesidad de información específica y encaminados a resolver problemas, a tomar de ci siones o a la in ves tigación.

Asimismo, se relacionan con esos patrones de bús que da, en tre otros, los pro veedores de información que son más usados para satisfacer esa necesidad de información, ${ }^{4}$ como pue den ser uni da des de información, vendedores de información, colegas, etcétera.

Esos patrones de búsqueda de información son diferentes entre los países de sarrollados y los subdesarrollados. ${ }^{5}$ Esto de bi do, como se se ña ló en pá rra fos an teriores, al contexto social que influye en las necesidades de información y por lo tan to en la ma ni fes ta ción de és tas a tra vés del comportamiento de la persona.

Un problema aparte, que indudablemente tam bién tie ne in fluen cia so bre el comportamiento del usuario, es el hecho de que su búsqueda de in for ma ción está deli mi ta da por los obs tácu los de ac ce sibilidad a la in for ma ción dis po ni ble en las bibliotecas, así como al uso de sus recursos, principalmente, en los países en vías de desarrollo.

El com por ta mien to en la bús que da de infor ma ción en tre los paí ses en vías de desarrollo y los desarrollados ha sido vislumbrado con base en cinco factores: sistemassociales, infraestructura, disponibilidad de literatura no convencional, factores administrativos y factores económicos. 6

Lo an te rior co rro bo ra que el fac tor so cial es de ter mi nan te en las nece si da des de información y por ende en el comportamiento informativo de los individuos.

Como se mencionó en párrafos anteriores el término necesidades de información parece abarcar también el comportamien to in for ma ti voy la sa tis fac ción de usua rios, por lo cual los en con tra mos íntimamente relacionados en diversos estudios que se han realizado.

Existen estudios, no propiamente sobre las necesi da des de in for ma ción, sino más bien, so bre la ma nifes ta ción de és tas, así te ne mos, por ejem plo, elcom por ta miento en la recolección de información de dos grupos (científicos y científicos sociales), se no ta ron las ob ser va cio nes y se lograronestablecerlas siguientescategorías: fuentes de información; uso y función de los ín di ces y abs tracts de re vis tas; aten ción y va lor a las con fe ren cias; de legación de la búsqueda de información; habilidades lingüísticas; y estímulos a la investigación. ${ }^{7}$

1 Pacho O’Donnel. Teoría y técnica de la psicoterapia grupal. — Buenos Aires : Amorrortu, 1974. — p. 40.

2 Pimrumpai Premsmit. "Information needs of academic medical scientists at Chulalongkorn University". - En: Bulletin of medical library association.- Vol. 74, no. 4, 1990. - p. 383.

3 O'Donnel, op. cit. p. 27.

4 Pimrumpai, op. cit., p. 383.

5 Idem, p. 385.

6 Girja Kumar. "Social science: a conceptual framworks". - En: The use of information in the changing world. — North Holland : Elsevier, 1984. - p. 245.

7 Idem. 


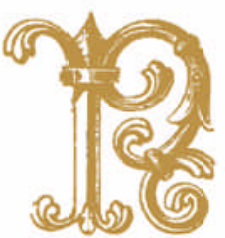

La existencia de necesidades de

información puede ser observada a través del comportamiento

informativo que tengan las

personas o, en caso concreto,

los usuarios de una biblioteca.

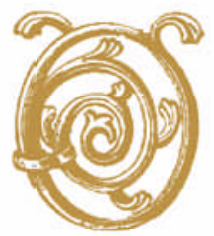

Otro ejem plo fue el observar los pa tro nes de comportamiento informativo de los científicos sociales, el cual indicó el uso de varias categorías de documentos: libros y revistas, principalmente, y otros documentos como: reportes, periódicos y documen tos nopublicados ${ }^{8}$ y literatura gris.

En otros estudios con los estudiantes de ciencias y tecnología, éstos presentaron ciertas manifestaciones de sus necesidades de información como la utilización de libros para el desarrollo de sus materias, o de ar tícu los de re vis tas cuan do necesitan información actualizada. ${ }^{9}$

Asi mis mo los cien tífi cos delárea de geología tienen patrones de búsqueda de información y utilizan ciertas técnicas como son: contactos personales (con cole gas), ba ses de da tos y uso de la bi blio teca. ${ }^{10}$

También los geofísicos para mantenerse actualizados utilizan: las redes personales (colegios invisibles); los contactos que en cuen tran en E-MAIL y newletter; el uso de revistas, colecciones personales de reimpresos; y bases de datos. ${ }^{11}$

Los patrones de búsqueda de información de los geólogos parecen tener una es ta bi li dad al paso de los años, y cam bian cuando el científico escala un nuevo trabajo en cuan to a sta tus y la dis po ni bi li dad del tiempo con que cuentan. ${ }^{12}$

Lo an te rior pa re ce in di car que al mo di ficar el contexto en el que trabajan estos científicos, sus necesidades de información cam bian y la ma ni fes ta ción de és tas a tra vés de su com por ta mien to in for ma tivo también se modifica. Pero parece haber ciertas similitudes cuando se trata de in di viduos per te ne cien tes a áreas ge ne rales más amplias, como la relación entre geólo gos y geo fí si cos, que son de la mis ma área, salvo por alguna diferencia.

También la realización de estudios sobre el com por ta mien to del usua rio en rela ción con los ser vi cios que le son pres ta- dos por los Servicios de Información, es mu chas ve ces lle va da a cabo con la es peran za de vol ver los más efi cien tes y ac cesibles, ${ }^{13}$ es pe ran do, tal vez, que iden ti fiquen las necesidades de información de los usuarios para poder satisfacerlas plenamente.

Entonces, la ma ni fes ta ción de las necesidades de información que tiene una persona se observará en el comportamiento que presente ésta, dicho comportamiento puede abarcar hábitos, costumbres, actitudes, procedimientos y habilidades, modos de acción, etcétera, que exhiba en la búsqueda de información y en el em pleo de las fuen tes y re cursos de in for ma ción; de igual ma ne ra este comportamientoinformativoestarárelacio na do con el con tex to en que se ubi que la persona.

En suma, la existenciadenecesidades de información puede ser observada a través delcom portamien to in for mativoque tengan las personas o, en caso concreto, los usuarios de una biblioteca.

\section{EL SURGIMIENTO DE LAS NECESIDADES DE INFORMACIÓN EN LOS INVESTIGADORES Y SU MANIFESTACIÓN}

Entre las funciones de la universidad se en cuen tra la in ves ti ga ción, ésta selle va a cabo en los cen tros e ins ti tu tos de in ves tiga ción o en al gu na otra uni dad con es tos fines.

Para lograr un avance científicoesnecesario que el investigador esté mejor informado, ya que la información es un factor importante para el progreso de la cien cia y ésta, a su vez, de sem pe ña un papel fundamental para la consecución de los objetivos sociales, económicos y políticos de un país. ${ }^{14}$

El personal académico que lleva a cabo estas labores en la universidad es el investigador (o en su caso, el profesor, dependiendo de la institución); este perso-

$8 \quad$ Ibidem, p. 248.

9 J. Day. "Information needs of science and technology students". — En:Education libraries bulletin. — Vol. 30, p. 1, Spring 1987. — p.

10 J. Bichteler. y Dederich Ward. "Information-seeking behavior of geoscientists". — En: Special libraries. - Vol. 80, no. 3 Winter 1989. - p. 170-171.

11 Ibidem, p. 173-174.

12 Ibidem, p. 176.

13 Ivonne Magaña Rodríguez. "El usuario y la información”. En: Boletín de información documental del sector comercio. - No. 1, ene.-feb. 1982 . - p. 43

14 Elsa Barberena. Las necesidades de información en el área científica. — [México] : [s.1., s.a.]. — p. 6. 
nal requiere de apoyo informativo para desempeñareficientementesuactividad.

Para apoyar la labor de investigación en lo referente a información se tiene que contar con una biblioteca u otra unidad de información que cumpla con este obje ti vo, por ci tar un ejem plo, en la Uni versidad Nacional Autónoma de México se cuenta con un Sistema Bibliotecarioque brinda este apoyo a la investigación.

Entonces, cada biblioteca, centro de documentación o centro de información que apoye esta labor, necesita conocer las necesidades de información de los académicosdedicadosalainvestigación.

Pero las obras que tra tan so bre este asunto (las ne ce si da des de in for ma ción de investigadores) son escasas y comprueban que to da vía no se sabe cómo es que un investigador resuelve sus necesidades de información, ${ }^{15}$ ni cuáles son éstas.

En el con tex to de otros paí ses, aun en tre las diversas áreas (humanidades, ciencias sociales y las científicas) es muy poco lo que co no ce mos acer ca de las necesidades de información de científicos sociales, por ejem plo, com para da con los científicos de las áreas duras. ${ }^{16}$ Asimismo son pocos los estudios que han sido desarrollados dentro de las necesidades de in for ma ción de los pro fe sio na les, y no precisamente investigadores en ciencias sociales. ${ }^{17}$

En realidad es poco lo que se ha escrito acer ca de las necesida des de in for ma ción que tienen los investigadores, más bien se han cen tra do los es tu dios en di feren tes aspectos como son los siguientes:

Recursos y fuentes de informaciónque utilizan tan to los hu ma nis tas como los científicos.

El tipo de fuente que usan, predominan te men te, como por ejem plo, el libro sobre la publicación periódica, por los humanis tas; y la publicación pe riódica sobre el libro, por los científicos.

Comportamientoinformativo, es decir, el com por ta mien to en la bús que da de la in for ma ción que ne ce si tan. Asícomo si de le gan esa ac ti vi dad a otros o la rea lizan ellos mismos.

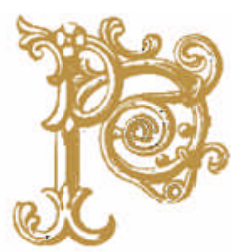

Son pocos los estudios que han sido desarrollados dentro de las necesidades de información de los profesionales, y no precisamente investigadores en ciencias sociales

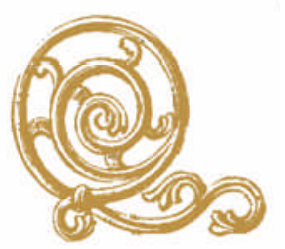

Idiomas en que buscan la información, así como idio mas en los que leen y/o citan las obras que consultan.

- Comportamiento en la manera en que usan las fuentes y recursos informativos, por ejemplo, si utilizan varias obras a la vez (con jun ti va) o de ma ne ra consecutiva (de una en una).

La edad de los materialesqueconsultano más bien delainfor maciónque necesitan.

Por lo an te rior, se pue de ob ser var que los diferentes es tu dios realizados es tán inclina dos ha cia la manifes ta ción de las necesidades de in for ma ción que tie nen di versos sec to res, es de cir, el com por ta mien to en la búsqueda de información, más no en conocer qué información necesitan.

Como se ha planteado en los apartados anteriores, el comportamiento en la búsqueda de información cae, en términos generales, dentro de las necesidades de información, pero sólo como manifestación de que és tas exis ten en los in ves ti gadores.

En este apar ta do se men cio na rán al gu nos estudios que tratan sobre la identificación de las necesidades de información (los cuales son muy pocos).

De en tre los es tu dios que se han rea li za do con los diversos grupos de personas sobre las ne ce si da des de in for ma ción - los cuales se refieren, no precisamente a inves ti ga do res, en di chas áreas; pero cu yos resultados pueden servir para ilustrar este trabajo-, se en cuen tran los realizados con biotecnólogos y profesionales del área de geofísica y geología.

Es necesario señalar que en los estudios realizados, específicamente del área cien tífi ca se pre sen tan las mis mas ca racterísticas señaladas en los párrafos anteriores (que se inclinan más al comportamiento informativo), salvo que en algunos estudios de Brasil, éstos sí logran determininar qué información es la que necesitan ciertos sectores, no solamen te el de los in ves ti ga do res, ya que dichos estudios no son exclusivos de este tipo de grupo.

Así tenemos, también, que aunque no se trata de investigadores, sino de estudiantes de las áreas de cien cia y tec no lo gía, en

15 Pablo Velázquez. Las necesidades de los usuarios de la información agrícola en América Latina . - [Buenos Aires : s.n., 1972]. — p. 5 .

16 N. G. Satish. Attitude toward information : a study of social scientists. - New Delhi, : Concept Publishing, $1994 .-$ p. 30.

17 Ibidem, p. 34. 
el es tu dio de Day se en con tró que di chos estudiantes presentaban necesidades de informacióntécnica y productiva para el desarrollo de gran parte de sus materias escolares. ${ }^{18}$ De esta forma se relacionan susnecesidadesconsus labores escolares. Lo encontrado en la escasa literatura sobre el tópico demuestra que en el área científica, la metodología para realizar los estudios sobre las necesidades de infor ma ción de los sec to res de geo cien cias y tecnología mineral, así como del área debiotecnología, es rigurosa, tanto en el diseño como en la selección de la muestra de estudio.

De entre los estudios, en el área científica, que des de el pun to de vis ta tra ta do en este documento, lograron determinar las necesidades de información del sector que investigaron se obtuvieron los siguientes resultados:

Los geólogos tienen necesidades de información según los siguientes tipos: ${ }^{19}$

Nuevos métodos y técnicas.

- Nuevos proyectos.

Estadísticas sobre reserva, producción y consumo mineral.

- Nuevos equipos y productos.

Calendario de congresos, encuentros, eventos, etcétera.

Legislación mineral.

Situación mineral en Brasil.

- Situación mineral en el extranjero.

- Información bibliográfica.

Los biotecnólogos tienen las siguientes necesidades de información: ${ }^{20}$

Se presentan divididas en tres áreas:

Biotecnólogos e instituciones del área de la salud:

Apelaciones generales en el área de la salud.

Detección de bacterias patógenas.

Aplicaciones en la industria

farmacéutica:

- Producción de antígenos parasitarios.

- Producción de antisueros monocionales.

- Producción de vacunas.

- Producción de enzimas.
Procesos enzimáticos.

Tecnología de fermentación.

Biotecnólogos e instituciones del área agropecuaria:

Aplicaciones generales en el área agropecuaria.

- Cultura de cuidado de plantas.

Control de plagas, bioinsecticidas.

Mejoramiento genético vegetal.

Nutrición vegetal:

- Función biológica del nitrógeno.

- Biofertilizantes.

- Inoculantes.

- Micorrizas.

Producción de vacunas (defensas animales).

Producción de enzimas (industria de alimentos).

Producción de levaduras (industria de alimentos).

Producción de proteínas unicelulares (industria de alimentos).

\section{Biotecnólogos del área de energía:}

Tecnología de fermentación.

- Fermentación alcohólica.

Procesos enzimáticos.

Aplicaciones generales de productos de energía:

- Ma te rias pri mas ener gé ti cas con énfasis en materias amiláceas, celuloicas, sacarinos, residuos urbanos e industriales.

- Producción de etanol.

- Producción de biogas.

- Reducción de polulantes

(contaminantes).

- Reducción de humo y otros contaminantes.

Equipos para procesos

biotecnológicos.

- Biodegradables.

- Fermentadores.

Asi mis moes tos gru pos de bio tec nó logos requieren de mayor información, la cual está resumida en los siguientes tipos:

- Información sobre cursos de capacitación de personal.

Noticias o datos sobre investigación básica y aplicada desarrollada en el país (Brasil) y en el extranjero.

Información sobre aplicaciones industriales de procesos biotecnológicos.
In for ma ción so bre con tra tos de com pra de tecnología efectuados por brasileños, abordando contenido, aplicación, condiciones, y sediciones de tecnología.

Información sobre el impacto de biotecnologías en el medio ambiente.

Análisis e impacto socieconómico de la biotecnología.

Información sobre legislación y regula cio nes téc ni cas de in te rés para elárea de biotecnología.

Planes y programas gubernamentales cuya implementación exige caer en el campo de la biotecnología, política de ciencia y tecnología.

Ad mi nis tra ción de la in ves ti ga ción y el desarrollo.

Información sobre los servicios técnico-científicos que ofrecen sobre biotecnología en Brasil.

Informaciónsobre la disponibilidad de consultoríasnacionales con sus respectivas áreas de competencia.

Información sobre la disponibilidad y características de bancos de germoplasmas y de microorganismos en el país (Brasil) y en el extranjero.

Bioinformática: programas aplicados para biotecnología.

Los científicos médicos académicos tienen tres grandes necesidades de información: ${ }^{21}$
Identificar información actualizada.
Estudios o datos relevantes.
Encontrar un tópico de investigación.

Por lo ex pues to en los párrafos an te riores de este apartado se puede notar que es poca la investigaciónque se ha realizado sobre las necesidades de información en lore la cio na do con su sur gi mien to e identificación en diversos sectores.

En cambio, se han escrito, en mayor número, diversoses tu dios so bre la manifestación de estas necesidades de información a través del comportamiento informativoenlabúsquedadeinformación. Algunos resultados de los estudios acerca del comportamiento informativo de va rios gru pos de per so nas en los sec to res

18 Day J., op. cit., p.4.

19 Departamento Nacional da Produçao Mineral. "Estudo da demanda de informaçao no sector de geociencia s e tecnologia mineral" — En: Ciencia da informaçao do Brasilia. - Vol. 15, no.1, Jan-Jun, 1986. - p.93.

20 Fundaçao da Tecnologia Industrial. "Estudo da demanda de informaçao dos usuarios da area de biotecnologia" — En: Ciencia da informaçao do Brasilia. - Vol. 15, no. 2, jul-dez, 1986. —p. 170.

21 Pimrumpai, op. cit., p. 385. 
de humanidades, ciencias sociales y científica, se mencionan a continuación.

\section{HUMA N I D A D E S Y CIENCIAS SOCI A L ES}

En el sec tor de las hu ma ni da des, los in dividuos (los humanistas y los científicos sociales) presentan determinadas características, como las siguientes:

a) Trabajan de forma individual, ${ }^{22}$ esta carac te rís tica escon sis ten te en gran parte de la literatura sobre el tema, un número de investigaciones parecen corroborar este he cho, pero qui zá esta carac te rís tica se deba a la misma actividad de trabajo de manejo de ideas o concepciones o de la creación imaginativa del hombre.

b) Poca conexión o relación con colegas de la misma área, ${ }^{23}$ parece, que como consecuen cia de un trabajo in di vidual, la existencia de colegios invisibles en el área de humanidades es escasa, puesto que no es pro mi nen te la exis ten cia de éstos para el intercambio de información.

Pero es ob vio que le dan im por tan cia al contacto personal con otra gente para un posible intercambio de ideas y de materiales. $^{24}$

El recurrir a colegas para obtener la información que necesitan no está dentro de las prime rasfuen tes utili za das por los profesionales en leyes, ${ }^{25}$ por ejemplo.

Los científicos sociales se enfrentan todavía a una laguna de comunicación entre ellos, ${ }^{26}$ ade más de que ca re cen de formación de los colegios invisibles.

Pero se debe mencionar que los investigadores sociales le dan cada vez más importancia a la dualidad de la co- municación oral y al uso de la literatura para obtener información. ${ }^{27}$

c) Disgusto por delegar la búsqueda de información; ${ }^{28}$ parece ser que los humanis tas pre fie ren rea li zarellos mis mos sus búsquedas de literatura sobre su requerimiento de información, posiblemente debido a que es difícil expresar en lenguaje conciso o en términos de indización los tópicos de su interés, ya que éstos se dan por el desarrollo de su propia actividad, por lo que se vuelven en algunos casos inteligibles o muy vagos, ya que sólo se tiene la idea de lo que andan buscando, mas no de una manera clara y concisa.

Son pocos los humanistas que tienen ayudantes para poder delegar la búsqueda de in for ma ción, por eso la ma yo ría de las veces ellos mismos la realizan. ${ }^{29}$

Los investigadores sociales no utilizan intermediarios que realicen las búsquedas de información que requieren, sino que lo hacen ellos mismos, ${ }^{30}$ pero todo pa re ce in di car que lo ha cen más fre cuentemente con la información corriente, es decir, delegan más veces la actividad de búsque dacuandoes re tros pectiva, ${ }^{31}$ esto también se debe a que el problema de la naturaleza de la terminología usada en las ciencias sociales es inestable, o sea, cambia continuamente. 32

d) Los humanis tas, pa re ce ser, tie nen una ten den cia a ho jear los li bros o fuen tes documentales que se encuentran en los estan tes de la bi blio te ca o ar chi vo para buscar información, aunque no se podría generalizar esta conducta. ${ }^{33}$ Otro tanto

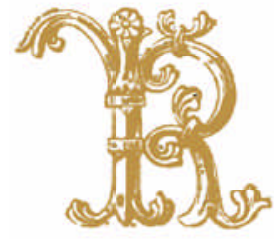

Los investigadores sociales no utilizan intermediarios que realicen las búsquedas de información que requieren, sino que lo hacen ellos mismos, pero delegan más veces la actividad de búsqueda cuando es retrospectiva

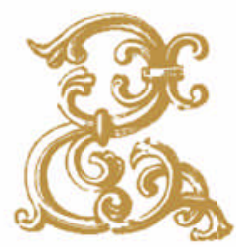

22 - C. Fulton. "Humanists as information users : a review of the literature". — En:Australian academic and research libraries. — Vol 22, no. 3. 1991, - p. 189.

- M.F. Stieg. "The information of needs of historians". — En: College and research libraries. — November $1981 .-$ p. 556.

23 - Fulton, op. cit., p. 189

- Stieg, op. cit., p. 556.

24 C. Corkill y M. Mann. Information needs in the humanities : two postal survey. — Sheffield: University of Sheffield, 1978. - p. 56.

25 M. S. Feliciano. "Acces to law : information needs of researchs in law and the public". — En: The use of information in the changing world. - North Holland : Elsevier, 1984. - p. 202.

26 M. Slater. "Information needs and communication problems of social scientists The United Kingdom situation". — En: International journal of information and library research. - Vol. 1, no. 2, 1989. - p. 134

27 M. Slater. Information needs of social scientists : a study by desk research and interview. - [London] : British Library Research, 1989.- p. 40 .

28 Idem.

29 Corkill, op. cit., p. 55.

30 Slater, "Information needs and communication...", op. cit., p. 135.

31 Slater, Information needs of social..., p. op. cit., 38.

32 Slater, "Information needs and communication...", op. cit., p. 135-137.

33 Fulton, op. cit., p. 190. 
ocurre con los investigadores sociales sobre la tendencia a hojear los materiales, para descubrir algo de información que les interese (serendipity). 34

Los profesionales en leyes tienden a utili zar como fuen te de in for ma ción para la investigación legal la biblioteca, ${ }^{35}$ lo mismo ocurre con algunos científicos sociales. ${ }^{36}$

e) Se dice que los humanistas tienden a usarmateriales primarios, ${ }^{37} \mathrm{o}$ de pri me ra mano, ma nus cri tos, li bros o do cu men tos originales, esto debido, posiblemente, también al desarrollo de su actividad. ${ }^{38}$ De igual manera, los científicos sociales utilizan los libros, manuscritos, te sis, reportes de in vesti ga ción, in for mes, planes y programas gu bernamen ta les pararealizar su trabajo. 39

El es tu dio de Stieg mues tra que los ma teriales más utilizados por los historiadores son los libros, las revistas y los manuscritos, 40 teniéndolos como de pri me ra manou ori gi na les, ya que se gún este es tu dio lo que me nos tien den a uti lizar son los microformatos.

f) Mucha de la literatura pone énfasis en que en los humanistas predomina el uso de la mono grafía para sus in ves ti gaciones, en contraste con las ciencias, donde el artículo de la revista es priorita- rio, ${ }^{41}$ aunque no se podría generalizar este hecho del todo, ya que muchos le dan la misma importancia. ${ }^{42}$ De igual manera los científicos sociales tienen preferencia por el libro y después por la publicación periódica, pero también utilizan los reportes de investigación, tesis, periódicos, publicaciones gubernamentales, estadísticas socioeconómicas,programas y planes de desarrollo nacional, informes de ministerios, departamentos, etcétera, y documentos con estadísticas de datos. ${ }^{43}$

Los profesionales en leyes tienden también a utilizar los libros como fuente para satisfacer sus necesidades de información. ${ }^{44}$

El es tu dio de Stieg mues tra que el for mato que predomina en los historiadores es el libro. ${ }^{45}$

Los cien tíficos so cia les re quie ren deluso de la li te ra tu ra gris, ${ }^{46}$ por lo cual es ne cesario que este tipo de fuentes esté disponible para ellos.

g) Los humanistas tien den a usar los materiales con una gran dispersión a través de las grandes divisiones del conocimiento, es decir, utilizan documentos que pertenecen no sólo al área de humanidades sino a otras áreas del conocimiento. ${ }^{47}$ Los humanistas utilizan una varie dad de títu los, peroesta variabilidad depende del tema investigado. ${ }^{48}$

h) La edad de los documentos en humani da des no es re le van te, es de cir, los materiales que se utilizan en esta área no pierden su valor con el tiempo, ya que las humanidadesnecesitanmaterialesretrospectivos. 49

La información en humanidades no es realmente buscada por su fecha. Mucha gente usa material retrospectivo como una fuen te pri ma ria o se cun da ria, pero no siempre es cierto, es obvio que otros investigadores necesitan materialreciente, aunque no lo necesitan por ser actual, sino por que ellos es tán tra ba jan do un periodo reciente y no retrospectivo. 50

Asimis mo para los cien tíficos so cia les la li te ra tu ra tien de a te ner un lar ga vida media, sobre todo para los materiales socio-históricos; la obsolescencia de los materiales es muy baja respecto a las ciencias naturales. ${ }^{51}$

i) El ma te rial en len gua ex tran je ra es usado y citado por los humanistas. Pero en los científicos sociales el lenguaje extranjero representa un problema, ya que al parecer no manejan idiomas fuera del inglés, a excepción del francés. ${ }^{52}$ Aunque los cien tífi cos so ciales tie nen ac ce so

34 Slater. "Social scientists' information needs in the 1980s".—En:Journal of documentation. — Vol. 44, no. 3, sep. 1988. — p. 232.

35 Feliciano, op. cit., p. 202.

36 Slater, "Information needs and communication...", op. cit., p. 137.

37 Fulton, op. cit., p. 191.

38 - D. O. Case. "The collection and use of information by some american historians : a study of motives and methods". — En: Library

Quaterly. - Vol. 61, no. 1, 1991. - p. 70

- Corkill, op. cit., p. 55.

39 S.P. Agrawal y M. Lal. "Information needs of social scientifics". — En:International library review. — no. 19, 1987. — p. $292-293$.

40 Stieg, op. cit., p. 551.

41 Fulton, op. cit., p. 191.

42 Corkill, op. cit., p. 55.

43 Agrawal, op. cit., p. 288-289.

44 Feliciano, op. cit., p. 202.

45 Stieg, op. cit., p. 551.

46 Slater, "Information needs and communication...". op. cit., p. 141-142.

47 Fulton, op. cit., p. 192.

48 Corkill, op. cit., p. 55.

49 Fulton, op. cit., p. 192.

50 Corkill, op. cit., p. 55.

51 Slater, “Information needs and communication...”, op. cit., p. 138.

52 - Slater, "Social..,". op. cit., p. 233.

- _ _, "Information needs and communication...", op. cit., p. 139-140. 
a las obras de los paí ses de ha bla in gle sa, uti li zan el servi cio de tra duc ción, pero no con mucha frecuencia. ${ }^{53}$ Este servicio predomina en los países desarrollados. En con tras te con lo an te rior, los his to riadores, en un alto porcentaje, no leen en diferen tes len guajes, se gún el es tu dio de Stieg. 54

j) En lo concerniente a herramientas bibliográficas, los es tu dios rea liza dos in di can que se tien de al uso de las bi blio grafías como elemento para descubrir información relevante; 55 los índices y abstracts son poco utilizados, posiblemente debido a lo que se men cio nó en la búsqueda de información, que es difícil ce ñir se a un len gua je, de bi do a que pa sar un tó pi co de in ves ti gación en hu manidades a términos de indización puede no llevar a la uniformidad deseada.

De esta for ma el uso de bi blio gra fías e índi ces y abs tracts fue va ria ble en di ver sos estudios realizados sobre humanistas. ${ }^{56}$ Los cien tífi cos so cia les tien den a uti li zar el servicio de elaboración de bibliografías sobre tópicos específicos. ${ }^{57}$

k) En el uso de bases de datos en línea, los humanistas presentan los inconvenientes de carecer de una terminología pre ci sa, el tras la dar las ideas y con cep tos a términos de búsqueda y la fal ta de unifor mi dad del soft ware no per mi te utili zar similares estrategias de búsqueda entre ellos. ${ }^{58}$ l) Entrelosinvestigadores socialesexiste la tendencia a utilizar otras bibliotecas y sus ser vi cios, apar te de la que les atien de a ellos directamente. ${ }^{59}$ Entre los servicios que más utilizan los historiadores podemos citar el préstamo interbibliotecario; 60 pero el uso de otras bi blio te cas y el préstamo interbibliotecario dependen del tema de investigación. ${ }^{61}$

El uso del préstamo interbibliotecario entre los es tu dian tes de arte y di se ño fue señalado como un medio para obtener la información que requerían para sus cursos, ${ }^{62}$ la cual no era cu bier ta en su to ta lidad por la biblioteca que los atendía.

m) Los investigadores sociales tien den a utilizar los materiales de manera consecutiva y conjuntiva, es decir, una gran parte de éstos lee los materiales uno tras otro; otra parte consulta varios materiales al mismo tiempo. ${ }^{63}$

Sin embargo, otros estudios indican que exis te un gran nú me ro de hu ma nis tas que uti li za una gran va rie dad de obras de manera conjunta al mismo tiempo. ${ }^{64}$

n) Los cien tífi cos so cia les pre sen tan una compleja necesidad de información, misma que no sólo se limita a impresos, sino a da tos, ar chi vos de da tos, como por ejem plo, un dato de un cen so, de una votación, de estadísticas; asimismo de archivos de datos legibles por máquina (bases de datos). ${ }^{65}$

\section{CIENTÍFICOS}

En el área de cien cias pu ras y aplicadas, los grupos de personas presentan también ciertas características, entre las que se encuentran las siguientes:

a) El re cur so de in for ma ción prin ci pal de los cien tífi cos son otros co le gas, es de cir, otros científicos (la comunicación e inter cam bio de in for ma ción con otros colegas), se hace a través de colegios invisibles. ${ }^{66}$ Asimismo en el rango de fuentes de información que también consideran importantes tenemos: la revista, los libros, los encuentros, los abstracts, los sistemas de recuperación de literatura, y las bibliografías. ${ }^{67}$

Tam bién los geólo gos con si de ran que su prin ci pal fuen te de in for ma ción son otros co le gas, así como la uti li za ción de las revistas, reimpresos, libros, catálogos, bases de datos bibliográficas y numéricas per so na les. Tam bién la uti li za ción de biblio te cas y cen tros de in for ma ción como fuentes de información en las cuales se puedan hojear las nuevas revistas, preguntar al bibliotecario directamente, utili zar el ca tá lo go en lí nea o en tar je tas, índices impresos, hojear libros de los estantes, te ner las ta blas de con te ni do de las revistas, servicios de bases de datos bibliográficas y numéricas. 68

b) Los geólo gos y es pe cia lis tas en tec nología mineral consideran importante los servicios que presta una biblioteca. ${ }^{69}$ Pero como re cur so para con se guir docut

53 Agrawal, op. cit., p. 292.

54 Stieg, op. cit., p. 556.

55 Ibidem., p. 554.

56 Corkill, op. cit., p. 55.

57 Agrawal, op. cit., p. 290.

58 Fulton, op. cit., p. 193.

59 Slater, "Social...". op. cit., p. 232

60 Stieg, op. cit., p. 553.

61 Corkill, op. cit., p. 55.

62 J. Day y E. MacDowell. "Information needs and use of art and design student". — En: Education libraries bulletin . — Vol. 28, p.1, Spring 1985. - p. 34

63 - Slater, "Social..." op. cit., p. 232.

- _ _ "Information needs and communication...," op. cit., p. 138.

- - —, Information needs of social..., op. cit., p. 51.

64 Corkill, op. cit., p. 55.

65 K. M. Heim. "Social scientific information needs for numerical data: the evolution of international data archive infraestructure". En: Collection management. - Vol 9, no. 1, Spring 1987. — p. 1-5.

66 Beberlee A. French. "User needs and library services" . - En: Library trends. — Vol. 38, no. 3 Winter, 1990. — p. 419.

67 Ibidem., p. 420.

68 J. Bichteler y Dederich W."Information-seeking behavior of geoscientists”. — En: Special libraries. — V ol. 80, no. 3 Winter 1989.—p. 170.

69 Departamento Nacional da Produçao Mineral, op. cit., p. 91. 
mentos, sigue pesando como fuente de información el recurrir a otros colegas (colegios invisibles).

c) El utilizar los servicios y documentos de otras bibliotecas es característico de los geólogos y tecnólogos minerales, 70 asi mis moelprés ta mo de documen tosentre colegas de la misma área. En el caso de los geólo gos aun que con si de ran len to el préstamo interbibliotecario lo utilizan para obtención de los documentos. ${ }^{71}$

d) Leen documentos técnicos en lengua ex tranjera. En tre los geólogos (de Bra sil) las len guas más co no ci das son el es pa ñol y el inglés, aunque se considera conveniente el pago de un servicio de traducción mediante la biblioteca, ya sea para traducción com ple tao versióncon den sada del texto. ${ }^{72}$ El servicio de traducción está in te gradoen los servi cios biblio tecarios y de información en los países desarrollados.

Los geólogos uti lizan preferen te men teel inglés y descartan otros lenguajes incluyendo los del tercer mundo, si requieren algo en otro idioma es factible el servicio de tra duc ción, aun que no es generalizado en to dos los cien tífi cos de geocien cias. ${ }^{73}$ d) Los biotecnólogos utilizan las siguientes fuentes primarias de información: memorias de congresos; preimpresos; revistas técnico-científicas; estados de arte; libros técnicos y de texto; literatura gris; patentes; te sis; ma nua les téc nicos; catálogos de productos y equipos; periódicosgenerales; y otras publicaciones de editoriales comerciales. ${ }^{74}$

e) Según unos estudios, los científicos en geociencias invierten mucho tiempo en la bús que da de in for ma ción- de 2 a 10 horas por semana. 75

Por lo ex pues to en los apar ta dos e in ci sos anteriores acerca de ambas áreas (humani da des y cien cias so cia les, y cien tífica), todo parece in di car que el área cien tífica es en la que se ha investigado acerca de cuál es la información que necesitan los científicos y que además se ha valido de la uti li zación de una me to do lo gíari gu ro- sa apli ca da en la rea li za ción de di chos estu dios, no así para los hu ma nis tas. Es así comose han ob te ni doal gu nos resultados donde se puedenidentificarconbastante claridad las necesidades de información del sector estudiado (científicos).

Por lo expuesto, hasta el momento, se puede observar que los investigadores tienen necesidades de in for ma ción y que hay que considerar que éstas pueden estar influenciadas por diversos factores como:

- Lugardonderealizansuinvestigación.

Área de investigación.

- Tema de investigación.

- Tipo de investigación.

Apoyos para realizar su investigación. Conocimientos y experiencia en el tema de investigación.

- Experiencia en la utilización de métodos de investigación.

- Uso de los recursos y fuentes informativas.

Hay que mencionar que existe otro elemen to que in flu ye en sus ne ce si da des de informacióny la manifestación deéstas.

Una vez satisfecha una nece si dad de información, surge otra, pero entre la presentación de una necesidad de información y la si guien te, exis te un fac tor que es el tiempo, es decir, al transcurrir del tiempo y ver satisfecha su necesidad de información, elinvestigador tendrá otra, posiblemente diferente o producto de la anterior. Entonces al transcurrir el tiem- po es como van surgiendo, manifestándo se y satis fa cién do se las ne ce si da des de información en el investigador.

Entonces se puede decir que el medio (o contexto) en el cual trabajan los investiga do res in flu ye en sus nece si da des de infor ma ción y en la ma ni fes ta ción de és tas a través de un comportamiento informati vo y que és tas van a cam biar al trans currir el tiempo.

\section{PROPUESTA DE UN MODELO SOBRE LAS NECESIDADES DE INFORMACIÓN DE LOS INVESTIGADORES}

Para tratar de comprender y apoyar una teoría acer ca de las ne ce si da des de in formación e intentar explicar cómo surgen las ne ce si da des de in for ma ción en los investigadores se puede proponer un modelo (en esta etapa, muy esquemático), que incluya los elementos que intervienen en dicho fenómeno.

En el esquema de la figura 1, se empieza abosquejarestefenómeno(véase Fig.1). Los elementos señalados en la figura 1 corresponderían a:

a) El surgimiento o aparición de una necesi dad de in for ma ción en el in vestigador.

b) La ne ce si dad de in for ma ción que tie ne elinvestigador la manifes taráa tra vés de uncomportamiento(comportamientoin-
Figura 1

Las necesidadesde informacióndelinvestigador

\begin{tabular}{|c|c|c|}
\hline $\begin{array}{l}\text { Necesidadesde } \\
\text { información del } \\
\text { investigador }\end{array}$ & $\begin{array}{l}\text { Comportamiento } \\
\text { informativo del } \\
\text { investigador }\end{array}$ & $\begin{array}{l}\text { Satisfacción de la } \\
\text { necesidad de informa- } \\
\text { ción del investigador }\end{array}$ \\
\hline
\end{tabular}

70 Ibidem., p. 91

71 Bichteler, op. cit., p. 172

72 -Departamento Nacional da Produçao Mineral, op. cit., p. 92-94.

- Fundaçao da Tecnologia Industrial., op cit., p. 178.

73 Bichteler, op. cit., p. 176.

74 Fundaçao da Tecnologia Industrial, op. cit., p. 172-175.

75 Bichteler, op. cit., p. 170 

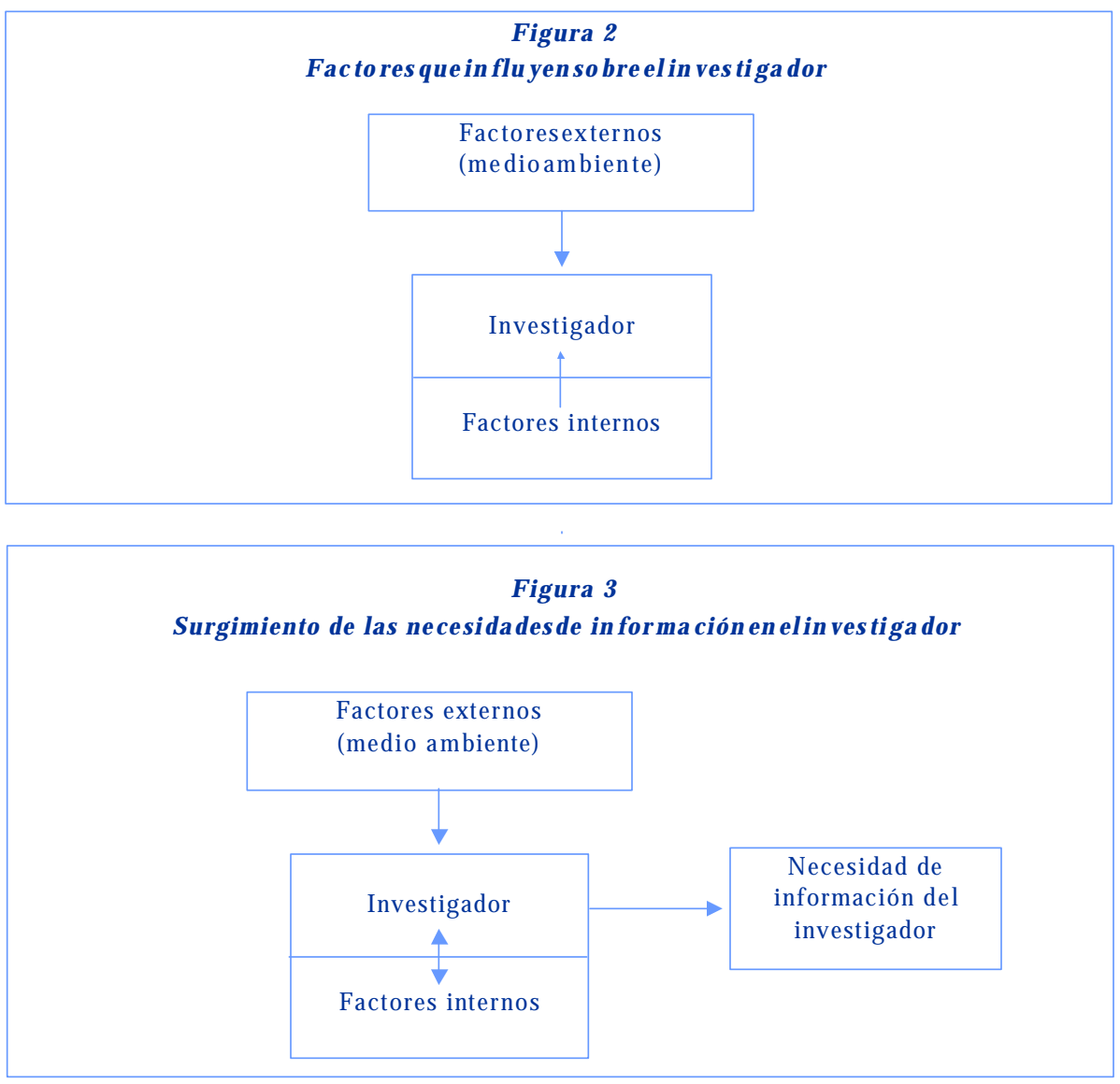

Figura 4

Manifestación de las necesidadesde informacióndelinvestigador

\begin{tabular}{|c|c|c|}
\hline $\begin{array}{c}\text { Necesidades de } \\
\text { información del } \\
\text { investigador }\end{array}$ & Se manifiesta a tra vés de un & $\begin{array}{c}\text { Comportamiento } \\
\text { informativo del } \\
\text { investigador }\end{array}$ \\
\cline { 2 - 3 }
\end{tabular}

formativo) para buscar la información que necesita.

c) Un ter cer ele men to que se ría la sa tisfac ción de esa ne ce si dad a tra vésde obtener la información requerida, a la cual llegará por medio de un comportamiento.

Por lo expresado en los apartados anterio res, la apa ri ción de las ne ce si da des de información está dada por la influencia de dos factores:

1.- Los internos: características propias del investigador, como serían: experiencia, conocimiento e información que po sea so bre el tema de su in ves tigación; métodos y técnicas que está inclinado a utilizar comúnmente en sus proyectos de in vestigación; recur- sos y fuen tes de in for ma ción que co múnmen te uti li za, así como su adies tra mien to en la utilización de los recursos bibliográficos y de información (índices, bibliográficas, bases de datos, etcétera). Características propias de su trabajo como in ves ti ga dor: línea, tema y eta pa de su in ves ti ga ción; mé to dos y téc ni cas que está empleando en su investigación y su labor de observador y cuestionador de los fenómenos que estudia y ocurren en su entorno.

2.- Los ex ternoso ambien ta les: ambien te laboral en donde realiza sus actividades: políticas y objetivos del centro, instituto o lugar donde realiza su trabajo; políticas generales de in vestiga- ción que maneje su institución; recursos económicos que se asignen para realizar su proyec to de in ves ti ga ción; tam bién se in clu yen en este fac tor el apo yo in for mativo a su investigación: servicios bibliotecarios y de información que le sean brindados. En ge ne ral se con si de ra todo el contexto en el cual se ubica su investigación.

Sólo se presentan algunos elementos inclui dos den tro de es tos dos fac to res, pero pueden existir algunos más.

Los dos factores anteriores afectan al investigador, por lo tanto influyen en las necesi da des de in for ma ción que pre sen te (véase Fig. 2).

Cuan do los dos fac to res afectan al in vesti gadoraldesarrollarsus acti vidadesacadémicas, entonces éste presenta una necesidad de información.

La ne ce si dad de in for ma ción del in ves tigador está bajo la influencia de ambos factores, el interno y el externo (véase Fig.3).

Cuan do el in ves ti ga dor tie ne una ne ce sidad de información la manifestará a través de un comportamiento informativo (véase Fig. 4).

También en los apartados anteriores se mencionó que el comportamiento informativo de los investigadores está bajo la influencia de ambos factores y en especial bajo el de los apoyos que tiene el investigador en relación con los servicios bibliotecarios y de in for ma ción que se le brindaban en su institución.

Entonces el comportamiento informativo está tambiéninfluenciado por los factores ambientales (ambiente laboral, en específico, los apoyos informativos), y lascaracterísticasindividuales delinvestigador -experiencia y conocimiento del tema, etcétera- (véase Fig. 5).

La manifestación de las necesidades de información a través del comportamiento informativo que presenta el investigador al bus car la in for ma ción que nece si ta loconduce asatisfacerdichanecesidad.

Una vez sa tis fe cha su ne ce si dad de in forma ción el in ves ti ga dor, al pa sar del tiempo, presentará otra.

El tiempo es un elemento que está presente desde la aparición de la necesidad de in for ma ción del in ves ti ga dor has ta su manifestación en un comportamiento y hasta la sa tis fac ción de la mis ma, ya que en otro momento dado del tiempo el in- 


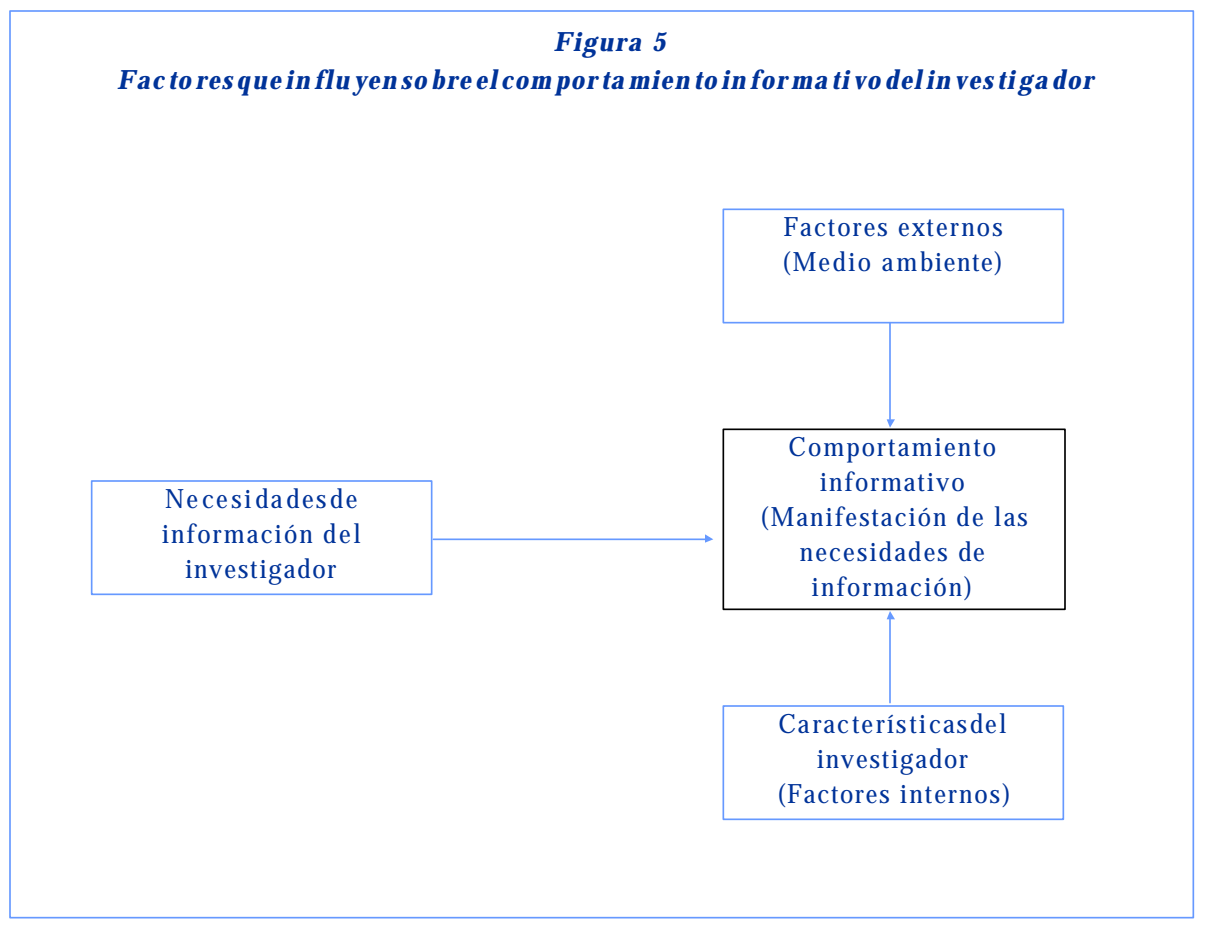

Figura 6

Surgimiento de las necesidades de información del investigador al trans currir el tiempo

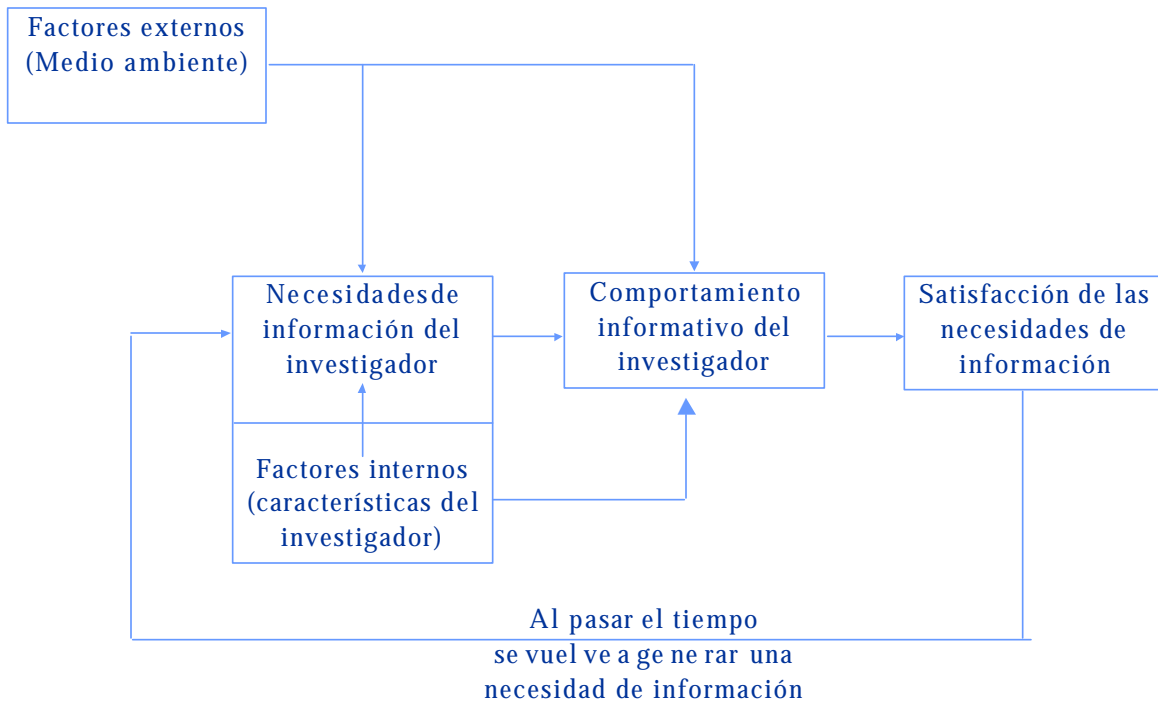

ves ti ga dor ya no ten drá la mis ma ne ce sidad de información, sino que aparecerá de la anterior o derivada de ella (véase Fig. 6).

\section{CONCLUSIONES}

A par tir de lo ex pues to en el de sa rro llo de este trabajo podemos concluir lo siguiente:

Losinvestigadorestienennecesidadesde información, las cuales serán manifestadas a tra vés de un com por ta mien to (comportamiento informativo) para buscar la información que satisfaga sus necesidades.

Las necesidades de información de los investigadores están influidas por diversos factores: internos y externos.

Entre los internos están las características del investigador (experiencia, conocimientos sobre el tema, etcétera), y su actividad como investigador (línea, tema, eta padelain ves ti gación, etcétera), en tre los externos podría mos men cionar el ambiente laboral (políticas de investigación, recursos económicos, etcétera). Los factores internos y ambientales con su gran mo vi li dad tie nen in fluen cia en el surgimiento de las necesidades de información de los investigadores; asimismo en la ma ni fes ta ción de és tas a tra vés de su comportamiento informativo.

Una vez satisfechas las necesidades de información de los investigadores y al paso del tiempo es como surgirán otras, ya sea originadas por las anteriores o completamente nuevas.

Entonces podemosobservar que el fenómeno que repetidamente presentan los investigadores está relacionado con, por lo me nos, dos fac to res - con sus res pecti vos ele men tos cada uno-: am bien ta les e internos, aña dien do otro ele men to más que sería el tiempo; dichos factores los podemos graficar en un esquematentativo (véase Fig. 6).

De esta ma ne rapode mos decirque exis ten prin ci pios que re gu lan la apa ri ción de las ne ce si da des de in for ma ción en los in vestigadores, lle ván do nos a la posibilidadde ha blar de la exis ten cia de una teo ría acerca de las necesidades de información. 


\section{BIBLIOGRAFÍA}

AGRAWAL, S.P. y M. Lal. "In for ma tion ne eds of so cial scien ti fics". — En:International libraryreview. - No. 19, 1987. - p. 287-299.

BARBERENA, Elsa. Las necesidades de información en el área científica. - [Mé xico] : [s.1., s.a.]. -20 p.

BICHTELER, J. y De de rich Ward. "In for ma tion-seeking beha vior of geos cien tists". En: Special libraries. — Vol. 80, no. 3 Winter 1989. — p. 169- 178.

BLYUNMENAU, D.I. "Cla rifying some ini tial con cepts in the theory of in for ma tion needs". - En: Nauchnoteknicheskayainformatsiya. — Se rie 2, no. 2, 1986. - p. 48- 57 (pá gi nas de la ver sión en in glés).

CASE, D. O. "The collection and use of information by some american historians : a study of motives and methods". - En: Library Quaterly. - Vol. 61, no. 1, 1991. - p. 61-82.

CORKILL, C. y M. Mann. Informationneeds in the humanities: two postalsurvey. She ffield : Uni ver sity of She ffield, 1978. - p. 56.

DANIELS SHEPARD, Ma riet ta. Planeamiento nacional de los servicios de información. - Wa shing ton D. C. : OEA, 1972.

DAY, J. "In for ma tion ne eds of scien ce and te chnology stu dents". — En:Edu ca tion libraries bulletin. - Vol. 30, p. 1, Spring 1987. - p. 1-6.

- - - y E. Mac Dowell. "In for ma tion ne eds and use of art and de sign stu dent". En: Education libraries bulletin. - Vol. 28, p.1, Spring 1985. - p. 34.

DURRACE, J . "In for ma tion need".- En:Rethin king the library information age. VII. - U.S. Office of Educational Research Goverment, office of library process, 1988 .

"Es tu do da de man da de in for maçao dos usuarios da area de bio tec nologia"/Fun daçao da Tec nologia In dus trial. - En:Cienciada informaçaodo Brasilia. — Vol. 15, no. 2, jul-dez, 1986. - p. 163-192.

"Estudo da de man da de in for maçao no sec tor de geo cien cias e tec no lo gia mi ne ral"/ De parta men to Na cional da Produçao Mineral. — En:Cien cia da in for maçao do Brasilia. - Vol. 15, no.1, Jan-Jun, 1986. - p.81-98.

FELICIANO, M.S. "Ac ces to law : in for ma tion ne eds of re searchs in law and the public". - En: The use of information in the chan ging world. - North Ho lland : Elsevier, 1984. - p. 197-208.

FRANTS, Va lery I. "The ne eds for in for ma tion and some as pects of in for ma tion re trieval : sys tem cons truc tion". En: Jour nal of the ame ri can so cietyfor in for mation science. - Vol. 39, no.2 Mar, 1988. - 86-91 p.

FUL TON, C. "Hu ma nists as in for ma tion users : a re view of the li te ra tu re". — En:Australian acade mic andre searchlibraries. — Vol. 22, no. 3. 1991, - p. 189. 
FRENCH, Beberlee A. "User needs and library servi ces". - En: Library trends. Vol. 38, no. 3 Win ter, 1990. - p. 419.

HEIM, K. M. "So cial scien ti fic in for ma tion ne eds for nu me ri cal data: the evolu tion of international data ar chi ve in fraes truc tu re". - En: Collection management. Vol 9, no. 1, Spring 1987. - p. 1-5.

KUMAR, Girja. "So cial scien ce: a con cep tual framworks". - En:The use of in for mation in the chan ging world. - North Holland: El se vier, 1984. - p. 241-251.

MAGAÑARODRÍGUEZ, Ivon ne. "El usua rio y la in for ma ción". En: Boletín de informacióndocumentaldel sectorcomercio. - no. 1, ene.-feb. 1982. - p. 42- 57.

MORALESCAMPOS, Es te la. "So cie dad e in for ma ción". - En: OMNIA : re vis ta de la Coordinación Generalde Estudios de Pos grado. — Año 6, no. 20, sep. 1990. p. 83-88.

O’DONNEL, Pacho. Teoría y técnica de la psicoterapia grupal. -Buenos Aires : Amorrortu, 1974.

PIMRUMPAIPREMS MIT . "Information needs of academic me dical scien tists at Chula longkorn Uni ver sity". - En: Bulle tin ofme dicallibraryassociation.- Vol. 74, no. 4, 1990. - p. 383-387.

RODRÍGUEZLUIS, Ira dia. "Es tu dio de las ne ce si da des de in for ma ción de los profe sio na les e in ves ti ga do res en el Sis te ma Na cio nal de Sa lud". — En:Acta de información científicaytécnica.-Vol. 23, no.1(150), 1990.—p.44-64.

SA TISH,N.G. Attitude towardinformation: a study of socialscientists. - New Delhi, : Concept Publishing, 1994.

SHERA, Jesse. Fundamentos delaeducaciónbibliotecológica. - México: UNAM, Centro Unversitario de Investigaciones Bibliotecológicas, 1990.

SLA TER, M. "In for mation needs and commu ni ca tion problems of social scien tists The United King dom si tua tion”. - En:Internationaljournal of information andibraryresearch. - Vol. 1, no. 2, 1989. - p. 134.

___ Information ne eds of so cialscien tists: a study bydeskresearchandinterview. — [Lon don] : Bro tish Li brary Re search, 1989.— p. 40.

-_- "So cial scien tists' in for ma tion ne eds in the 1980s".-En: Journal of do cumentation. - Vol. 44, no. 3, Sep. 1988. - p. 232.

STIEG, M F. "The in for ma tion of ne eds of his to rians". - En: Colle ge and re search libraries. - November 1981. - p. 55.

VELÁZQUEZ, Pa blo. Las ne ce si da des de los usua rios de la in for ma ción agrícola en AméricaLatina. - [Buenos Aires: s.n., 1972]. 